\title{
Physiology of chronic lung allograft dysfunction: back to the future?
}

\author{
Allan R. Glanville \\ Affiliation: The Lung Transplant Unit, St. Vincent's Hospital, Sydney, NSW, Australia.
}

Correspondence: Allan R Glanville MBBS, MD, FRACP, The Lung Transplant Unit, Xavier 4, St. Vincent's Hospital, Victoria Street, Darlinghurst NSW, Australia 2010. E-mail: allan.glanvilledasvha.org.au

@ERSpublications

Phenotyping chronic lung allograft dysfunction using lung volume measurements allows stratification of outcomes http://ow.ly/CF0Q309hx9Q

Cite this article as: Glanville AR. Physiology of chronic lung allograft dysfunction: back to the future? Eur Respir J 2017; 49: 1700187 [https://doi.org/10.1183/13993003.00187-2017].

Chronic lung allograft dysfunction (CLAD) is, in one sense, a term without a true consensus definition, although its common usage in the field of lung transplantation implies a base understanding throughout the community that it describes a lung allograft that does not work (well) [1]. There appears to be general agreement that CLAD most commonly occurs in a time-dependent fashion after transplant and is a harbinger of a foreshortened survival [2]. However, different phenotypes have been described based on a combination of physiological and radiological features [3]. A restrictive ventilatory defect, with or without interstitial pulmonary infiltrates and/or pleural thickening, has the worst prognosis and is also associated with inferior results from attempts at salvage by retransplantation [4]. One curious feature of the natural history of CLAD is the chameleon-like property of morphing from one predominant phenotype to another [5]. Perhaps this bespeaks our simplistic understanding of the root causative mechanisms, but emphasises there is still much to learn about this dominant complication of lung transplantation, which is the major risk factor for death in those who survive the perioperative period. The article by KNEIDINGER et al. [6] in this issue of the European Respiratory Journal attempts to fine-tune our insight into CLAD phenotypes and provides valuable information regarding the prognostic value of gas trapping in CLAD. Once again, the precision of pulmonary physiology assessment as a longitudinal tool is shown to provide a useful guide to survival. In this way, repeated measures of a safe and noninvasive test can assist individual patient management by highlighting early trends. Whether effective therapies are currently available is another question, but at least some stratification of those individuals less likely to deteriorate can be carried out. One perennial problem in this type of long-term retrospective single-centre analysis is the potential for variable adherence to testing schedules and the relatively small number of patients in each group. In this case, 20 had restrictive physiology (defined by a 10\% reduction in total lung capacity (TLC) from baseline) and 21 had gas trapping (defined as a residual volume/TLC $\geqslant 50 \%$ ) at CLAD onset. Both of these phenotypes had inferior survival compared with other patients with CLAD who predominantly had bronchiolitis obliterans syndrome (BOS), as defined recently in the Journal [7]. Is this surprising? Although the finding of a poor outcome with gas trapping has not been widely reported previously after lung transplantation, it makes biological sense and resonates with conventional knowledge that accepts that gas trapping is associated with an increased risk of dynamic hyperinflation (an increase in TLC) and thereby an increase in mechanical load on the inspiratory muscles, reduced mechanical advantage and increased oxygen cost of breathing [8]. The result is impaired exercise performance and greater levels of dyspnoea. Ultimately, respiratory cachexia and hypercapnoeic respiratory failure develop, with death commonly from respiratory tract infection. Conversely, there is now a building body of evidence regarding

Received: Jan 262017 | Accepted: Jan 262017

Conflict of interest: None declared.

Copyright OERS 2017 
the adverse prognosis of restrictive CLAD - whether associated with pleuroparenchymal disease or not. Upper lobe fibrosis was first described by Konen et al. [9] in 2003, and later, restrictive allograft syndrome (RAS) was described by SATO et al. [10], combining changes in TLC with computed tomography (CT) evidence to examine outcomes. A number of other authors have employed different physiological criteria of restriction, not always measuring lung volumes, and broadly found similar outcomes [11]. There are nuances of course, and while a case can be made for the expedience of measuring lung mechanics alone (spirometrically determined variables) to assess likelihood of the presence of a restrictive defect, it is refreshing to see the diligence of the current report, where full lung function testing was performed at least every 3 months using body plethysmography - recognised as the preferred laboratory tool for assessing lung volumes, as opposed to helium dilution, which may underestimate trapped gas and TLC.

All groups cannot afford such rigorous serial evaluation, or so it would seem, but weighed against the cost of CT scanning and bronchoscopy, perhaps a favourable cost-utility ratio could be determined.

Why is it so? What causes CLAD and are there different mechanisms for each phenotype? Likely there are, and we do have some specific outcomes. Both timing and severity of the development appear prognostically important and therein lies a clue (or two). As does distribution [12]. We know that severe primary graft dysfunction is a significant risk factor, as is cellular rejection, particularly lymphocytic bronchiolitis, but also antibody-mediated rejection - both HLA-related and associated with autoantibodies although the full picture is only beginning to emerge [13-17]. Even the air that we breathe is important, let alone community-acquired viral infection [18-22]. Perhaps matching therapies to individual recipients may one day provide a tailored approach with greater efficacy as there remains a firm conviction that one size does not fit all. It is always possible that some commonly employed immune suppressive therapies may be profibrotic in some individuals with specific genetic polymorphisms, and this may in part explain the variable incidence of restrictive physiology reported throughout the globe [23].

How should we incorporate the knowledge gained from the current study? In practice, it is a reminder of what we always knew. A deep understanding of pulmonary physiology with sensitive pattern recognition informs function of the lung, and perhaps nowhere else do we have such a predictable and compressed model of lung dysfunction to examine prospectively. The gauntlet has been thrown down as a challenge to us all to rethink our somewhat antediluvian approach to lung physiology of the pulmonary allograft and develop specific approaches to specific phenotypes based solidly on mechanistic principles, thereby availing our patients of the panoply of diagnostic and therapeutic strategies available at a stage where stabilisation may be associated with acceptable quality-of-life outcomes.

\section{References}

1 Glanville AR. Bronchoscopic monitoring after lung transplantation. Semin Respir Crit Care Med 2010; 31: 208-221.

2 Verleden GM, Raghu G, Meyer KC, et al. A new classification system for chronic lung allograft dysfunction. J Heart Lung Transplant 2014; 33: 127-133.

3 Verleden SE, Ruttens D, Vandermeulen E, et al. Restrictive chronic lung allograft dysfunction: where are we now? J Heart Lung Transplant 2015; 34: 625-630.

4 Verleden SE, Ruttens D, Vandermeulen E, et al. Predictors of survival in restrictive chronic lung allograft dysfunction after lung transplantation. J Heart Lung Transplant 2016; 35: 1078-1084.

5 Verleden SE, de Jong PA, Ruttens D, et al. Functional and computed tomographic evolution and survival of restrictive allograft syndrome after lung transplantation. J Heart Lung Transplant 2014; 33: 270-277.

6 Kneidinger N, Milger K, Janitza S, et al. Lung volumes predict survival in patients with chronic lung allograft dysfunction. Eur Respir J 2017; 49: 1601315.

7 Meyer KC, Raghu G, Verleden GM, et al. An international ISHLT/ATS/ERS clinical practice guideline: diagnosis and management of bronchiolitis obliterans syndrome. Eur Respir J 2014; 44: 1479-1503.

8 Gibson GJ. Pulmonary hyperinflation a clinical overview. Eur Respir J 1996; 9: 2640-2649.

9 Konen E, Weisbrod GL, Pakhale S, et al. Fibrosis of the upper lobes: a newly identified late-onset complication after lung transplantation? AJR Am J Roentgenol 2003; 181: 1539-1543.

10 Sato M, Waddell TK, Wagnetz U, et al. Restrictive allograft syndrome (ras): a novel form of chronic lung allograft dysfunction. J Heart Lung Transplant 2011; 30: 735-742.

11 Todd JL, Jain R, Pavlisko EN, et al. Impact of forced vital capacity loss on survival after the onset of chronic lung allograft dysfunction. Am J Respir Crit Care Med 2014; 189: 159-166.

12 Belloli EA, Wang X, Murray S, et al. Longitudinal forced vital capacity monitoring as a prognostic adjunct after lung transplantation. Am J Respir Crit Care Med 2015; 192: 209-218.

13 Christie JD, Bavaria JE, Palevsky HI, et al. Primary graft failure following lung transplantation. Chest 1998; 114: 51-60.

14 Huang HJ, Yusen RD, Meyers BF, et al. Late primary graft dysfunction after lung transplantation and bronchiolitis obliterans syndrome. Am J Transplant 2008; 8: 2454-2462.

15 Glanville AR, Aboyoun CL, Havryk A, et al. Severity of lymphocytic bronchiolitis predicts long-term outcome after lung transplantation. Am J Respir Crit Care Med 2008; 177: 1033-1040.

16 Levine DJ, Glanville AR, Aboyoun C, et al. Antibody-mediated rejection of the lung: a consensus report of the international society for heart and lung transplantation. J Heart Lung Transplant 2016; 35: 397-406.

17 Hachem RR, Tiriveedhi V, Patterson GA, et al. Antibodies to K-a 1 tubulin and collagen V are associated with chronic rejection after lung transplantation. Am J Transplant 2012; 12: 2164-2171. 
18 Verleden S, Scheers $\mathrm{H}$, Nawrot $\mathrm{T}$, et al. Lymphocytic bronchiolitis after lung transplantation is associated with daily changes in air pollution. Am J Transplant 2012; 12: 1831-1838.

19 Nawrot TS, Vos R, Jacobs L, et al. The impact of traffic air pollution on bronchiolitis obliterans syndrome and mortality after lung transplantation. Thorax 2011; 66: 748-754.

20 Glanville AR. Community-acquired respiratory viruses after lung transplantation: common, sometimes silent, potentially lethal. Thorax 2014; 69: 1-2.

21 Sayah DM, Koff JL, Leard LE, et al. Rhinovirus and other respiratory viruses exert different effects on lung allograft function that are not mediated through acute rejection. Clin Transplant 2013; 27: E64-E71.

22 Weinberg A, Lyu DM, Li S, et al. Incidence and morbidity of human metapneumovirus and other community-acquired respiratory viruses in lung transplant recipients. Transpl Infect Dis 2010; 12: 330-335.

23 Hostettler KE, Roth M, Burgess JK, et al. Cyclosporine A mediates fibroproliferation through epithelial cells. Transplantation 2004; 77: 1886-1893. 